\title{
ORIGEM E DISTRIBUIÇÃO INTRAPARENQUIMAL DA ARTÉRIA HEPÁTICA DO PREÁ (Galea spixii Wagler, 1831)
}

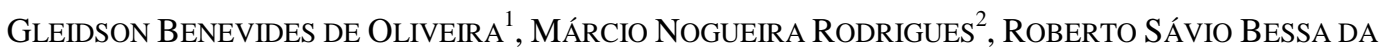 \\ Silva $^{1}$, Valéria Veras de Paula ${ }^{3}$, Maria ACelina Martins CarValho ${ }^{4}$, Moacir Franco de \\ OLIVEIRA $^{3}$
}

\begin{abstract}
${ }^{1}$ Pós-Graduandos da Universidade Federal Rural do Semi Árido, Mossoró, RN, Brasil. - gleidson_benevides@hotmail.com ${ }^{2}$ Pós-Graduando da Faculdade de Medicina Veterinária e Zootecnia da Universidade de São Paulo, São Paulo, SP, Brasil.

${ }^{3}$ Professores Doutores da Universidade Federal Rural do Semi Árido, Mossoró, RN, Brasil.

${ }^{4}$ Professora Doutora da Universidade Federal do Piauí, Teresina, PI, Brasil.
\end{abstract}

RESUMO

\begin{abstract}
O preá é um roedor pertencente à família Cavidae. O objetivo deste trabalho, considerando a inexistência de informações na literatura, foi descrever a origem e distribuição intraparenquimal da artéria hepática do preá. Foram utilizados 20 animais que morreram por causas naturais no Centro de Multiplicação de Animais Silvestres, Mossoró-RN, Brasil. Estes foram incisados medianamente e a aorta torácica injetada com solução de látex ou vinil. Os animais perfundidos com látex foram fixados em formol 10\% e dissecados após 72 horas. Os perfundidos com vinil tiveram o fígado removido e imerso em solução de $\mathrm{H}_{2} \mathrm{SO}_{4} 30 \%$ até completa corrosão do seu parênquima. Observou-se que a artéria hepática origina-se
\end{abstract}

da artéria celíaca e, ao longo de seu trajeto em direção ao fígado, emite a artéria gastroduodenal e posteriormente a artéria gástrica direita, continuando-se como artéria hepática própria. Esta se divide em ramos esquerdo e direito, ou emite ainda um ramo intermédio. O ramo esquerdo apresentou-se como tronco comum aos vasos destinados aos lobos lateral esquerdo, medial esquerdo e quadrado. O ramo direito da artéria hepática emitia ramos aos lobos lateral direito, medial direito e caudado. $\mathrm{O}$ padrão de origem e distribuição intraparenquimal da artéria hepática em preás assemelha-se ao de outros roedores.

PALAVRAS-CHAVE: artéria hepática; fígado; Galea spixii; roedor.

\section{INTRAPARENCHYMAL ORIGIN AND DISTRIBUITION OF THE HEPATIC ARTERY OF THE GALEA (Galea spixii Wagler, 1831)}

\section{ABSTRACT}

The Galea is a rodent from the Cavidae family. The aim of this study was to describe the intraparenchymal origin and distribuition of the galea hepatic artery considering the inexistence of information in the literature. Animals $(\mathrm{n}=20)$ from Centro de Multiplicação de Animais Silvestres Mossoró-RN Brazil were evaluated after dying by natural causes. The animals were incised medially and the thoracic aorta was cannulated for injection of latex or vinyl. The animals perfused with latex were fixed in formaldehyde $10 \%$ and dissected after 72 hours. The ones perfused with vinyl acetate had their liver removed and immersed in a $30 \% \mathrm{H}_{2} \mathrm{SO}_{4}$ solution until the complete corrosion of its parenchyma. It was observed that the hepatic artery in galea originates from the celiac artery and, along the way toward the liver, it sends off the gastroduodenal artery and then the right gastric artery, continuing as proper hepatic artery. This one is divided into right and left branches or sends off an intermediate branch. The left branch proved to be a common trunk to the vessels destined to the left lateral, left medial and square lobes. The right branch of the hepatic artery sends off branches to right lateral, right medial and caudate 
lobes. The origin and distribution patterns of the intraparenchymal hepatic artery in galea is similar to other

KEYWORDS: Galea spixii; hepatic artery; liver; rodent.

\section{INTRODUÇÃO}

O preá silvestre do semi-árido (Galea spixii Wagler, 1831) é um roedor pertencente à família Caviidae, sendo o mamífero mais abundante da caatinga. Pode ser encontrado na região nordeste do Brasil e nos estados de Goiás, Mato Grosso e Minas Gerais. Possui o corpo alongado, coloração uniforme, com a superfície dorsal cinza-escura e ventre branco. A cabeça e os olhos são grandes, as orelhas são curtas e arredondadas. Tem hábito crepuscular, vive em bandos e alimenta-se de folhas, ramos e frutos de plantas rasteiras, raízes tubérculos e casca de árvores jovens. O período de gestação deste roedor é de 50 dias e o número de filhotes, por parto, oscila de um a quatro, raramente mais (MENDES, 1987).

Segundo KÖNIG \& LIEBICH (2004), o suprimento sanguíneo do fígado é particularmente abundante, sendo a artéria hepática um ramo da artéria celíaca que supre o fígado com sangue rico em oxigênio. Os ramos da artéria hepática que penetram isoladamente no fígado são artérias terminais, que se ramificam à semelhança da veia porta, suprindo o parênquima e terminando nos sinusóides hepáticos, onde os fluxos sanguíneos se misturam.

Descrevendo a ramificação e distribuição da artéria celíaca em cutias, CARVALHO et al. (1994) afirmaram que artéria hepática é um ramo da artéria celíaca, assim como as artérias esplênica e gástrica esquerda. A artéria hepática emite, no seu trajeto em direção ao fígado, dois ramos colaterais, a artéria gástrica direita e um tronco comum às artérias pancreaticoduodenal cranial e gastroepiplóica direita, ou emite a artéria pancreaticoduodenal cranial e um tronco comum às artérias gástrica direita $\mathrm{e}$ gastroepiplóica direita.

$\mathrm{O}$ estudo da morfologia em animais selvagens é sempre uma fonte crescente de informações, especialmente quando se trata de espécies que apresentam algum potencial zootécnico ou valor biológico, como é o caso do Galea spixii, roedor da fauna nordestina com grande importância social, uma vez que sua carne é utilizada como fonte de proteína animal em comunidades carentes, ou mesmo por hábitos culturais do nordestino. Ressaltase ainda que o emprego sistemático de animais na experimentação é importante para o desenvolvimento de pesquisas de interesse médico rodents.

para a população humana, o que implica o conhecimento de morfologia, motivo pelo qual decidiu-se estudar a vascularização hepática do preá, sobretudo pela importância do fígado na biotransformação de fármacos. Dessa forma, a presente investigação científica teve como objetivos descrever a origem e a distribuição intraparenquimal da artéria hepática no preá, de modo a estabelecer um padrão para a espécie, bem como fornecer dados à anatomia comparada.

\section{MATERIAL E MÉTODOS}

No desenvolvimento deste estudo foram utilizados 20 preás machos adultos, que vieram a óbito por causas naturais e se encontravam em freezer no Centro de Multiplicação de Animais Silvestres da Universidade Federal Rural do SemiÁrido (CEMAS/UFERSA), licenciado junto ao IBAMA como criadouro cientifico (Registro 1478912). Ressalta-se que, em virtude da indisponibilidade de fêmeas, optou-se pela utilização somente de machos.

Os animais foram descongelados e logo incisados medianamente para abertura das cavidades torácica e abdominal, sendo que, em 10 preás, a aorta torácica foi canulada no sentido crânio-caudal para administração de solução de Neoprene látex "650" (Du Pont do Brasil S.A.) corado com pigmento vermelho (Globo S.A. Tintas e Pigmentos). Em seguida, esses animais foram imersos em solução aquosa de formol a 10\%, e após 72 horas da fixação, dissecados. Os demais animais foram perfundidos com solução de acetato de vinil corado de vermelho através da aorta torácica, sendo imediatamente o fígado removido e imerso em solução de ácido sulfúrico a $30 \%$ para obtenção de moldes vasculares.

$\mathrm{O}$ estudo baseou-se na nomenclatura adotada pelo INTERNATIONAL COMMITTEE ON VETERINARY GROSS ANATOMICAL NOMENCLATURE (2005) para a denominação das estruturas identificadas e os resultados comparados com a literatura referente a estudos com roedores e outros mamíferos.

\section{RESULTADOS E DISCUSSÃO}

A artéria hepática no preá origina-se a partir da artéria celíaca e, ao longo de seu trajeto em direção ao fígado, emite dois ramos colaterais, 
emitindo um tronco comum às artérias a artéria gástrica direita, continuando como artéria gastroepiplóica direita e pancreaticoduodenal cranial, hepática própria (Figura 1). denominada artéria gastroduodenal, e posteriormente

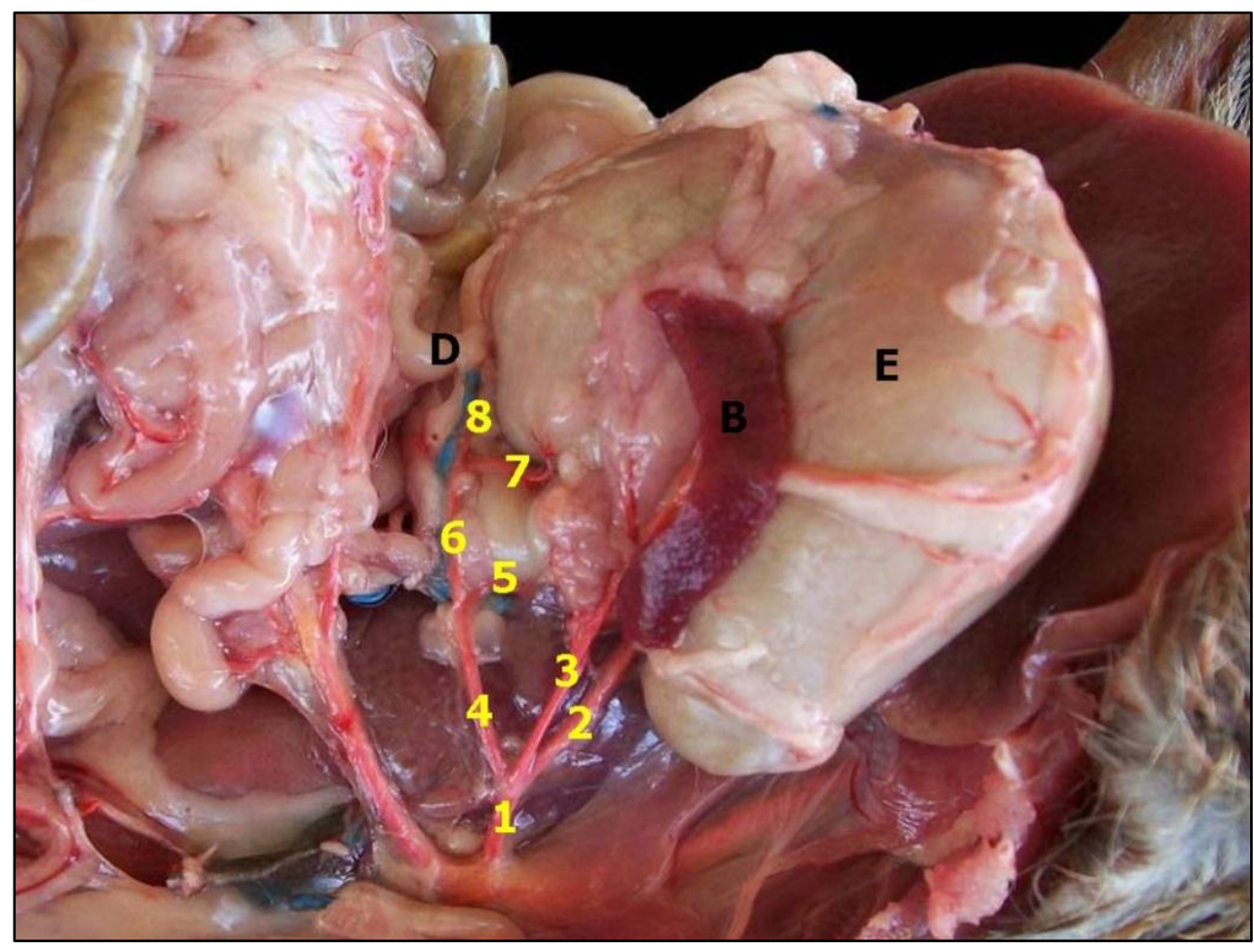

Figura 1- Fotografia da origem da artéria hepática do preá. Observa-se a artéria celíaca (1) e seus ramos, as artérias gástrica esquerda (2), esplênica ou lienal (3) e a artéria hepática (4). Tem-se ainda a artéria gastroduodenal (6), originando as artérias gastroepiplóica direita (7) e pancreaticoduodenal cranial (8) e a artéria hepática própria (5). Estômago (E), Duodeno (D), Baço (B). Mossoró, RN, 2009.

As dissecações do material injetado com látex Neoprene 650 evidenciaram que, em oito animais $(80 \%)$, a artéria hepática própria, antes de penetrar no fígado, emitiu ramos císticos, sendo que em sete desses emitiu um único ramo (Figura 2B) e, em apenas um animal, originou dois ramos. Ao atingir a fissura portal dividiu-se, mais frequentemente, em ramos direito e esquerdo (80\%) ou emitiu um terceiro ramo, o ramo intermédio, que se distribuiu em direção ao lobo medial direito $(20 \%)$ (Figuras 2B e C).

$\mathrm{O}$ ramo esquerdo apresentou-se como um tronco comum aos vasos destinados aos lobos lateral esquerdo, medial esquerdo e quadrado em $100 \%$ das observações. Verificou-se, ainda, que o ramo esquerdo em um dos moldes vasculares emitiu, além desses ramos, um ramo para o processo papilar do lobo caudado. Num segundo molde, o ramo principal esquerdo emitiu um ramo para a vesícula biliar. Em oito moldes analisados o ramo direito da artéria hepática emitiu ramos aos lobos lateral direito, medial direito e caudado (processo papilar e caudado) (Figura 2B). Em um molde, o mesmo emitiu ramos aos lobos lateral direito e lobo caudado (processo papilar e caudado) (Figura 2C) e outro emitiu ramos aos lobos lateral direito, processo caudado e vesícula biliar (Figura 2D).

No preá, a artéria hepática, juntamente com as artérias lienal e gástrica esquerda, é ramo da artéria celíaca, semelhante ao que descreve GETTY (1981) e KÖNIG \& LIEBICH (2004) em animais domésticos. 


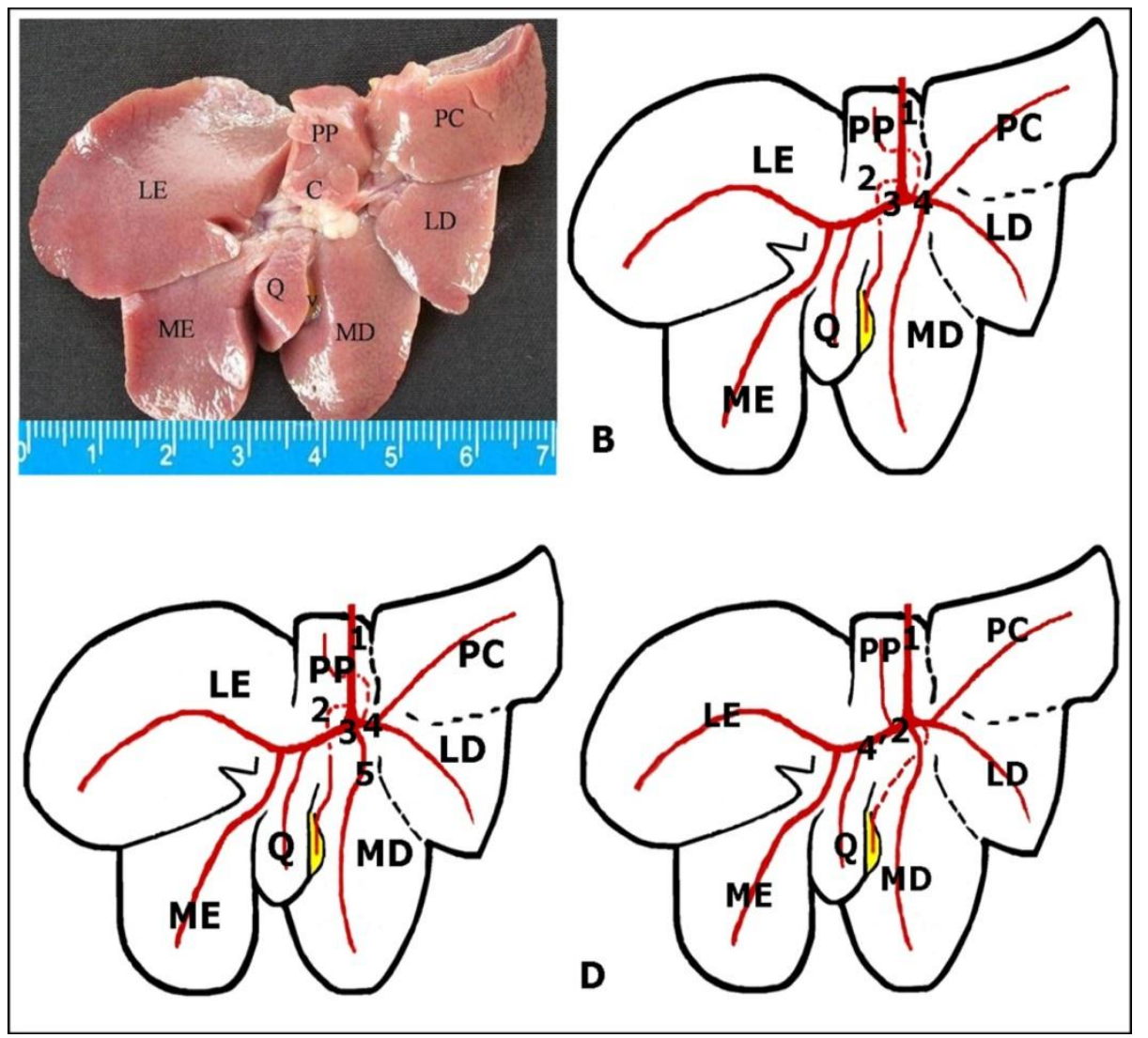

Figura 2- Fotografia da lobação do fígado e representação esquemática de seus moldes arteriais. Em A, a porção visceral do fígado, com seus lobos laterais direito (LD) e esquerdo (LE), mediais direito (MD) e esquerdo (ME), quadrado (Q) e lobo caudado com seus processos caudado (PC) e papilar (PP). EM B, observa-se o ramo cístico (2) e a divisão da artéria hepática (1) em ramos esquerdo (3) e direito (4). O direito emite ramos aos lobos lateral e medial direito (LD e MD) e processos papilar e caudado do lobo caudado (PP e PC) e o ramo esquerdo aos lobos lateral e medial esquerdo (LE e ME) e quadrado (Q). Em C, a divisão da artéria em ramos esquerdo (3), direito (4) e intermédio (5). Em D, o ramo direito emite ramos aos lobos lateral direito (LD), processo caudado (PC) e à vesícula (seta) e o esquerdo emite o tronco comum (4') e um ramo para o processo papilar (3'). Mossoró, RN, 2009.

SAMPAIO et al. (2001), estudando a distribuição da artéria celíaca em coelhos, afirmam que a artéria hepática própria origina-se mais frequentemente da artéria hepática, porém os autores chamam a atenção ao fato de a mesma originar-se da artéria gástrica direita ou da mesentérica cranial.

CARVALHO et al. (1994), descrevendo a artéria hepática em cutia, relataram que essa é ramo da artéria celíaca e que, ao longo do seu trajeto para o fígado, emite dois ramos colaterais, correspondentes à artéria gástrica direita e um tronco comum às artérias gastroepiplóica direita e pancreaticoduodenal cranial, da mesma forma que o observado no preá.

No Galea spixii, a artéria hepática emitiu até dois ramos císticos e apenas um ramo para o fígado, que se constituiu na artéria hepática própria e ao atingir o fígado ramificou-se em dois ou três ramos. MACHADO et al. (2002), estudando o ratão do banhado, mencionam que a artéria hepática originase de dois a quatro ramos císticos e penetra no fígado por meio de três a cinco ramos, diferentemente do observado no preá. Já COOPER \& SCHILLER (1975), no porquinho-da-índia, e AZEVÊDO et al. (2008), em trabalhos com cutia, verificaram que a artéria hepática divide-se em dois ramos principais, que se distribuem para os lobos direito e esquerdo, igualmente ao observado no preá, embora tenha sido observada a presença de um ramo intermédio nesse último estudo.

SOUZA et al. (2007) constataram que a artéria hepática na capivara divide-se mais frequentemente em dois ramos, direito e esquerdo, ao inserir-se no fígado, ou ainda podendo trifurcar-se nos ramos direito, intermédio e esquerdo.

AZEVÊDO et al. (2008) observaram, na cutia, que o ramo direito da artéria hepática é responsável pela vascularização dos lobos medial 
direito, lateral direito e caudado, enquanto o ramo esquerdo apresenta-se como tronco comum aos vasos que se destinam aos lobos medial esquerdo, lateral esquerdo e quadrado, podendo enviar ramos para o lobo caudado ao lobo lateral direito. No preá, no entanto, o lobo lateral direito em nenhum momento recebeu vasos oriundos do ramo esquerdo, limitando-se ao ramo direito da artéria hepática própria.

Em capivaras, o ramo esquerdo da artéria hepática emite vasos aos lobos lateral esquerdo, medial esquerdo, quadrado e medial direito, podendo ainda emitir ramos aos lobos lateral direito e caudado (processo papilar e caudado). Os lobos medial direito, lateral direito e caudado estão vascularizados por ramos oriundos do ramo direito. $\mathrm{O}$ ramo intermédio emite ramos aos lobos medial direito, quadrado e medial esquerdo ou esta representado pela artéria cística. Já o ramo direito destina-se aos lobos lateral direito e caudado, podendo emitir ramos aos lobos lateral esquerdo, medial esquerdo, quadrado e à vesícula biliar (SOUZA et al. (2007). No preá, o ramo intermédio direcionou-se somente ao lobo medial direito, enquanto, que os lobos lateral esquerdo, medial esquerdo e quadrado, em nenhum momento, receberam ramos oriundos do ramo principal direito da artéria hepática.

\section{CONCLUSÃO}

O padrão de origem e distribuição intraparenquimal da artéria hepática em preás assemelha-se ao de outros roedores, considerando a literatura consultada.

A exemplo do que se observa em outras estruturas de roedores, o padrão de distribuição intraparenquimal da artéria hepática do preá variou dentro da mesma espécie e entre animais machos.

\section{REFERÊNCIAS}

D. J. A.; MACHADO, G. V.; SOUZA, A. A. R.; XAVIER F. G. Distribuição intraparenquimal da artéria hepática em cutias (Dasyprocta sp, Rodentia). Brazilian Journal Veterinary Research Animal Science, v.45, n.1, p.5-10, $2008 . \quad$ Disponível em: <http://www.fumvet.com.br/novo/revista/45/n1/0510.pdf $>$. Acesso em 10 out. 2009.

CARVALHO, M. A. M.; MIGLINO, M. A.; DIDIO, L. J. A. Ramificação e distribuição da artéria celíaca na cutia (Dasyprocta aguti). Brazilian Journal Veterinary Research Animal Science, v.31, n.3/4, p. 191-197, 1994.

COOPER, G.; SCHILLER, A. L. Anatomy of the guinea pig. Cambridge: Harvard University Press, 1975. 417p.

GETTY, R. Anatomia dos animais domésticos. 5. ed. v.1. Rio de Janeiro: Guanabara Koogan, 1981. 1134p.

INTERNATIONAL COMMITTEE ON VETERINARY GROSS ANATOMICAL NOMENCLATURE. Nomina anatomica veterinária. 5. ed. Knoxville: World Association on Veterinary Anatomist, 2005. 190 p.

KÖNIG, H. E.; LIEBICH, H.G. Anatomia dos Animais Domésticos: texto e atlas colorido. v.2. Porto Alegre: Atmed, 2004. 399p.

MACHADO, G. V.; SOUZA, J. R.; GONÇALVES, P. R.; PARIZZI A.; DONIN, D. G. A. Artéria celíaca e seus ramos no ratão-do-banhado (Myocastor coypus Rodentia: Mammalia). Biotemas. v.15, n.2, p. 41-54, $2002 . \quad$ Disponível em: $<$ http://www.biotemas.ufsc.br/volumes/pdf/restaurados/15 2/41-54.pdf>. Acesso em 05 out. 2009.

MENDES, B. V. Plantas e animais para o Nordeste. Rio de Janeiro: Editora Globo, 1987. 167p.

SAMPAIO, M. A. P.; SAMPAIO, B. P. S.; DANTAS, A. C.; MANGIA, S. H. Distribuição da artéria celíaca em coelhos. In: CONGRESSO BRASILEIRO DE MEDICINA VETERINÁRIA (CONBRAVET), 28, 2001. Anais..., Salvador: 2001. p.57

SOUZA, W. M.; SOUZA, N. T. M.; CARVALHO, R. G.; CORREA, C. N. Topografia da artéria hepática no fígado de capivara. Ciência Rural. v.37, n.1, p. 141-145, 2007. Disponível em: 〈http://www.scielo.br/pdf/cr/v37n1/a22v37n1.pdf >.

AZEVÊDO, L. M.; CARVALHO, M. A. M.; MENEZES, Acesso em 20 nov. 2009. 